\title{
Altered gut microbiota correlate with different immune responses to HAART in HIV-infected individuals
}

Yirui Xie ${ }^{1 *}$ DD, Jia Sun ${ }^{1,2}$, Li Wei $^{1}$, Haiyin Jiang ${ }^{1}$, Caiqin Hu${ }^{1}$, Jiezuan Yang ${ }^{1}$, Ying Huang ${ }^{1}$, Bing Ruan ${ }^{1}$ and Biao Zhu ${ }^{1 *}$

\begin{abstract}
Background: Although gut microbiota dysbiosis has been reported in HIV infected individuals recently, the relationship between the gut microbiota and immune activation in patients with different immune responses to highly active antiretroviral therapy (HAART) is still not well understood. Gut microbiota and immune activation were studied in 36 non-HIV-infected subjects (healthy controls) and 58 HIV-infected individuals, including 28 immunological responders (IR) and 30 immunological non-responders (INR) ( $\geq 500$ and $<200$ CD4+ T-cell counts/ $\mu$ l after 2 years of HIV-1 viral suppression respectively) without comorbidities.
\end{abstract}

Results: Metagenome sequencing revealed that HIV-infected immunological responders and immunological nonresponders could not recover completely from the gut microbiota dysbiosis. At a 97\% similarity level, the relative abundances of Fusobacterium, Ruminococcus gnavus and Megamonas were greater, whereas Faecalibacterium, Alistipes, Bifidobacterium, Eubacterium rectale and Roseburia were more depleted in the IR and INR groups than those in the healthy controls. Ruminococcaceae and Alistipes were positively correlated with nadir and current CD4+ T-cell counts, but negatively correlated with CD8 + CD57+ T-cell counts. Inflammation markers and translocation biomarkers (LPS) levels were positively correlated with the abundances of genera Ruminococcus and Fusobacterium but were negatively correlated with the genus Faecalibacterium. The relative abundances of Escherichia-Shigella and Blautia were significantly higher in the IR than those in the INR group. Escherichia-Shigella were negatively correlated with the CD4/CD8 ratio but positively correlated with the amount of CD8 + CD57+ T-cells. Roseburia and Blautia were negatively associated with nadir CD4+ T-cell and positively associated with CD8 + CD57+ T-cell counts.

Conclusions: Gut microbiota dysbiosis may be one of the factors contributing to different immune responses and treatment outcomes to HAART.

Keywords: HIV-1, Gut microbiota, Immune activation, Immunological responders, Immunological non-responders, HAART

\footnotetext{
* Correspondence: 1312019@zju.edu.cn; zhubiao1207@zju.edu.cn

${ }^{1}$ State Key Laboratory for Diagnosis and Treatment of Infectious Diseases, National Clinical Research Center for Infectious Diseases, Collaborative Innovation Center for Diagnosis and Treatment of Infectious Diseases, The Department of Infectious Diseases, The First Affiliated Hospital, School of Medicine, Zhejiang University, 79, QingChun Road, Hangzhou 310003, China Full list of author information is available at the end of the article
}

(C) The Author(s). 2021 Open Access This article is licensed under a Creative Commons Attribution 4.0 International License, which permits use, sharing, adaptation, distribution and reproduction in any medium or format, as long as you give appropriate credit to the original author(s) and the source, provide a link to the Creative Commons licence, and indicate if changes were made. The images or other third party material in this article are included in the article's Creative Commons licence, unless indicated otherwise in a credit line to the material. If material is not included in the article's Creative Commons licence and your intended use is not permitted by statutory regulation or exceeds the permitted use, you will need to obtain permission directly from the copyright holder. To view a copy of this licence, visit http://creativecommons.org/licenses/by/4.0/ The Creative Commons Public Domain Dedication waiver (http://creativecommons.org/publicdomain/zero/1.0/) applies to the data made available in this article, unless otherwise stated in a credit line to the data. 


\section{Background}

Life expectancy of individuals infected with human immunodeficiency virus (HIV) has increased enormously and HIV infection has become a chronic disease that is manageable in the combination antiretroviral therapy (ART) era. Most patients receiving ART can achieve a distinct reduction of HIV viral load and improvement of CD4+ T-cell counts compared with nadir CD4+ T-cell counts (the patient's lowest CD4+ T-cell counts). However, the extent of immunological recovery varies greatly between individuals. The HIV-infected individuals who fail to achieve normalization of CD4+ T-cell counts despite persistent virological suppression are considered as immunological non-responders (INR) [1], which are in contrast with immunological responders (IR) [2]. As there is no unified definition of INR, the prevalence of INR ranges from 15 to $30 \%$ in the ART cohorts [3-5]. The INR were defined as patients whose absolute value of CD4+ T cells counts were less than 200 cells $/ \mu \mathrm{l}$ after years of ART, although 350 cells/ $\mu l$ were also used as a cutoff value in the literature [6]. In contrast, IR were defined as patients whose CD4+ T-cell counts were greater than 500 cells $/ \mu \mathrm{l}$ after receiving ART for years.

Emerging evidence suggests that the gut microbiome of HIV-infected patients are different from that of HIVuninfected individuals [7]. However, these studies have not considered the immune response to ART and there have been little research focusing on patients in China [7]. Recently, several studies have reported that gut microbiota is associated with $\mathrm{CD} 4+\mathrm{T}$-cell recovery in HIV-infected patients [8-10]. The microbiome of chronic HIV infected individuals was studied in China. However, these patients had heterogeneous HIV progressions and immune responses to ART [11, 12].
Studies showed that HIV-mediated destruction of gut mucosa could lead to local and systemic inflammation [13]. Moreover, chronic inflammation was reported to be associated with the gut microbiome in the non-AIDS population [14]. These studies suggest that the gut microbiome may play a part in the immune activation in HIV-infected individuals with ART [13, 15-17]. Despite many studies of the microbiome in HIV-infected patients, there have been relatively few reports discussing the gut microbiome that occur in patients with different immune responses to ART $[18,19]$. Therefore, a comparative study of the gut microbiome and immune activation was conducted on HIV patients with different immune responses to ART and the results are presented in this study. 16S ribosomal RNA (rRNA) targeted sequencing and flow cytometry were used to characterize the gut microbiome and their relationship with immune activation among immunodiscordant and immunoconcordant patients with long-term suppressive ART.

\section{Results}

Clinical characteristics and pyrosequencing data summary The characteristics of all 28 IR patients, 30 INR patients and 36 healthy controls, including demographics, clinical characteristics, and pyrosequencing results are presented in Table 1. There is no significant difference between the rate of the transmission route in the IR and INR groups $(p=0.779)$. The rate of homosexual (MSM) transmission route is $57.1 \%$ vs $51.7 \%$ in the INR and IR groups, whereas the rate of the heterosexual transmission route is $20.7 \%$ vs $21.4 \%$, and other rates are missing from their records. The viral load of all HIV-infected individuals with ART is not detected. Nadir and current $\mathrm{CD} 4+\mathrm{T}$ cell counts are significantly higher in the IR

Table 1 Clinical characteristics data summary

\begin{tabular}{|c|c|c|c|c|}
\hline & \multirow[b]{2}{*}{ Health Control } & \multicolumn{2}{|l|}{ HIV ART (+) } & \multirow[t]{2}{*}{$P$-value } \\
\hline & & Immune Responders (IR) & Immune Non-responders (INR) & \\
\hline Number of subjects & 36 & 28 & 30 & \\
\hline Gender male/female & $33 / 3$ & $25 / 3$ & $29 / 1$ & \\
\hline Age (mean \pm SD) & $33.11 \pm 3.95$ & $36.64 \pm 10.2$ & $36.6 \pm 7.19$ & NS \\
\hline $\mathrm{BMI}($ mean $\pm \mathrm{SD})$ & $21.42 \pm 3.27$ & $21.06 \pm 2.37$ & $20.67 \pm 2.74$ & NS \\
\hline Smoking & 1 & 0 & 1 & NS \\
\hline \multicolumn{5}{|l|}{ Transmission, no. } \\
\hline Heterosexual & NA & 6 & 6 & NS \\
\hline Homosexual transmission & NA & 16 & 15 & NS \\
\hline Data Missing & NA & 6 & 8 & NS \\
\hline HAART months (mean \pm SD) & NA & $37.25 \pm 13.61$ & $34.00 \pm 10.24$ & NS \\
\hline \multicolumn{5}{|c|}{ Ongoing cART regimen, no. patients (\%) } \\
\hline NNRTI based & NA & 25 & 27 & NS \\
\hline PI based & NA & 3 & 3 & NS \\
\hline
\end{tabular}

$N A$ (not available), NS (no significant) indicates $p$-value $>0.05$. NNRTI Non-nucleoside reverse transcriptase inhibitors, $P$ Protease inhibitor 
group than the INR group (Table 2). No differences in the duration of ART and ongoing ART medications are observed between the IR and the INR groups. Other characteristics such as gender, age and body mass index (BMI) are generally matched among the IR, INR and healthy controls. 3,549,077 high-quality sequences in total were obtained (average sequence length $440 \mathrm{bp}$ ) from 94 participants. Thirty-eight thousand eight hundred forty-nine sequences per sample on average were obtained from the healthy controls, while 35,947 and 38, 134 sequences per sample were obtained respectively from the IR and INR patients. Rarefaction was conducted on the OTU (Operational taxonomic unit) table to 30,174 reads per sample to avoid methodological artefacts. Specifically, 609 OTUs are defined in the healthy controls, while 486 OTUs and 567 OTUs in the IR and INR groups are defined relatively at a $97 \%$ similarity level. Significant differences of bacterial diversity (Shannon, Simpson, and Sobs), richness (ACE, Chao1) and Good's coverage are observed among the three groups, while no significant difference are found between the IR and the INR groups. A summary is shown in Table 2.

Table 2 Cellular immune activation markers sequencing data summary

\begin{tabular}{|c|c|c|c|c|}
\hline \multirow[t]{2}{*}{$\mathrm{T}$ cell markers } & \multirow[b]{2}{*}{ Health Control } & \multicolumn{2}{|l|}{ HIV ART (+) } & \multirow[t]{2}{*}{$P$-value } \\
\hline & & Immune Responders (IR) & Immune Non-responders (INR) & \\
\hline Nadir CD4 ${ }^{+} \mathrm{T}$ cells $($ mean $\pm \mathrm{SD})$ & NA & $309.89 \pm 128.81$ & $95.23 \pm 108.92$ & $<0.0001$ \\
\hline Current CD4 ${ }^{+} T$ cells (mean \pm SD) & NA & $608.30 \pm 158.25$ & $230.5 \pm 87.50$ & $<0.0001$ \\
\hline Current CD4+/CD8+ T-cell ratio & NA & $0.8 \pm 0.36$ & $0.35 \pm 0.20$ & $<0.0001$ \\
\hline HIV RNA & NA & ND & ND & \\
\hline \%CD4 + HLADR+CD38+ & NA & $7.72 \pm 4.30$ & $10.30 \pm 10.45$ & NS \\
\hline$\% C D 4+C D 25+$ & NA & $1.26 \pm 0.99$ & $1.09 \pm 1.04$ & NS \\
\hline$\% C D 4+C D 57+$ & NA & $2.57 \pm 1.92$ & $2.43 \pm 3.30$ & NS \\
\hline$\% C D 8+\mathrm{HLADR}+\mathrm{CD} 38+$ & NA & $20.68 \pm 11.35$ & $23 \pm 12.39$ & NS \\
\hline$\% C D 8+C D 57+$ & NA & $14.36 \pm 7.11$ & $23.98 \pm 12.30$ & 0.001 \\
\hline \multicolumn{5}{|l|}{ Cytokines } \\
\hline $\mathrm{IL}-2(\mathrm{pg} / \mathrm{mL}$, mean $\pm \mathrm{SD})$ & $9.47 \pm 6.79$ & $120.96 \pm 113.96$ & $153.81 \pm 118.79$ & $<0.0001$ \\
\hline $\mathrm{IL}-4(\mathrm{pg} / \mathrm{mL}$, mean $\pm \mathrm{SD})$ & $13.49 \pm 31.01$ & $34.52 \pm 43.30$ & $32.51 \pm 31.17$ & 0.009 \\
\hline IL-6(pg/mL, mean $\pm \mathrm{SD})$ & $8.39 \pm 12.57$ & $126.90 \pm 95.33$ & $130.82 \pm 60.62$ & $<0.0001$ \\
\hline IL-9(pg/mL, mean $\pm \mathrm{SD})$ & $3.68 \pm 6.20$ & $94.00 \pm 78.07$ & $96.57 \pm 48.71$ & $<0.0001$ \\
\hline IL10(pg/mL, mean \pm SD) & $2.48 \pm 2.35$ & $44.69 \pm 37.66$ & $51.32 \pm 31.65$ & $<0.0001$ \\
\hline IL-13(pg/mL, mean \pm SD) & $85.45 \pm 65.02$ & $96.76 \pm 71.54$ & $82.94 \pm 62.96$ & NS \\
\hline IL-17A (pg/mL, mean \pm SD) & $26.22 \pm 52.89$ & $133.41 \pm 161.61$ & $94.80 \pm 83.84$ & $<0.0001$ \\
\hline IL-17F $(\mathrm{pg} / \mathrm{mL}$, mean $\pm \mathrm{SD})$ & $5.45 \pm 5.68$ & $19.15 \pm 19.48$ & $20.59 \pm 20.34$ & $<0.0001$ \\
\hline $\mathrm{IL}-21(\mathrm{pg} / \mathrm{mL}$, mean $\pm \mathrm{SD})$ & $41.33 \pm 48.87$ & $104.43 \pm 88.11$ & $93.84 \pm 56.89$ & 0.002 \\
\hline $\mathrm{IL}-22(\mathrm{pg} / \mathrm{mL}$, mean $\pm \mathrm{SD})$ & $153.98 \pm 97.15$ & $291.60 \pm 176.36$ & $293.10 \pm 125.37$ & 0.001 \\
\hline IFN- $\gamma(\mathrm{pg} / \mathrm{mL}$, mean $\pm \mathrm{SD})$ & $12.55 \pm 29.52$ & $81.57 \pm 84.50$ & $69.36 \pm 83.42$ & $<0.0001$ \\
\hline TNF-a $(p g / m L$, mean \pm SD) & $8.39 \pm 15.57$ & $38.86 \pm 44.19$ & $32.22 \pm 26.47$ & $<0.0001$ \\
\hline LPS (pg/mL, mean \pm SD) & $24.22 \pm 18.88$ & $76.55 \pm 40.05$ & $104.98 \pm 56.15$ & $<0.0001^{\mathrm{b}}$ \\
\hline $\mathrm{sCD} 14(\mathrm{pg} / \mathrm{mL}$, mean $\pm \mathrm{SD})$ & $1583.60 \pm 292.80$ & $2480.42 \pm 999.88$ & $2142.65 \pm 496.53$ & $<0.0001$ \\
\hline \multicolumn{5}{|l|}{ Pyrosequencing data } \\
\hline Sobs index ${ }^{a}$ & $194.11 \pm 47.45$ & $116.89 \pm 39.71$ & $118.11 \pm 46.96$ & $<0.0001$ \\
\hline Shannon index ${ }^{a}$ & $3.14 \pm 0.53$ & $2.47 \pm 0.54$ & $2.44 \pm 0.56$ & $<0.0001$ \\
\hline Simpson index ${ }^{a}$ & $0.12 \pm 0.09$ & $0.19 \pm 0.11$ & $0.19 \pm 0.13$ & $<0.0001$ \\
\hline $\mathrm{ACE}^{\mathrm{a}}$ & $223.97 \pm 53.59$ & $147.26 \pm 39.98$ & $148.41 \pm 49.99$ & $<0.0001$ \\
\hline Chao 1 index ${ }^{a}$ & $224.29 \pm 54.76$ & $144.52 \pm 45.25$ & $145.37 \pm 51.01$ & $<0.0001$ \\
\hline Good's coverage (\%) ${ }^{a}$ & $99.82 \pm 0.04$ & $99.87 \pm 0.04$ & $99.87 \pm 0.04$ & $<0.0001$ \\
\hline
\end{tabular}

andicates that the alpha diversity was calculated after the reads number of each sample were equalized. NA (not available), ND (not detected), NS (no significant) indicates $p$-value $>0.05,{ }^{\text {b }}$ compared between the IR and the INR group 


\section{Compositional analysis of fecal microbiota}

Principal coordinate analysis (PCoA) by weighted UniFrac matrices shows obvious differentiation of bacterial communities between the IR and the healthy controls (PERMANOVA, pseudo-F: 8.99, $R^{2}=0.13, P=0.001$, Fig. 1a), the INR and the healthy controls (PERM ANOVA, pseudo-F: $8.77, R^{2}=0.12, P=0.001$, Fig. $1 \mathrm{~b}$ ), while no significant differences are observed between the IR and INR groups (PERMANOVA, pseudo-F: 0.80, $R^{2}=0.01, P=0.71$, Fig. 1 c).

The data of average relative abundances of each bacterial phyla and genera in patients and the healthy controls are showed respectively in the figures (Fig. 2; S1, S2 and S3 Fig) and Table (S1 Table). The Wilcoxon rank sum test was used to detect taxa with significant differences in relative abundances among groups (confidence interval method). At the phylum level, Bacteroidetes, Actinobacteria, Tenericutes and Lentisphaerae are more abundant in the healthy controls than in the IR group.
The relative abundances of Proteobacteria, Fusobacteria and Saccharibacteria are significantly higher in the IR group (S1 Fig. 2a) than those in the healthy controls. The relative abundances of 11 families are significantly different between the IR and the healthy controls. The relative abundances of 93 genera, including 15 predominant ( $>1 \%$ of the total sequences in either group) and 78 less-predominant genera, are significantly different between the healthy controls and the IR groups. Among the different predominant genera, Lachnoclostridium, Megasphaera, Escherichia-Shigella, Veillonella, Streptococcus, Fusobacterium, and Ruminococcus gnavus are found to be overrepresented in the IR group. The relative abundances of Faecalibacterium, Eubacterium rectale, Alistipes, Subdoligranulum, Bifidobacterium, Roseburia, Ruminococcaceae and Parasutterella are higher in the healthy controls (Fig. 2 and S1 Fig. 2b) than in the IR group. A taxonomybased bacterial comparison was conducted to define the differences between the healthy controls and the INR
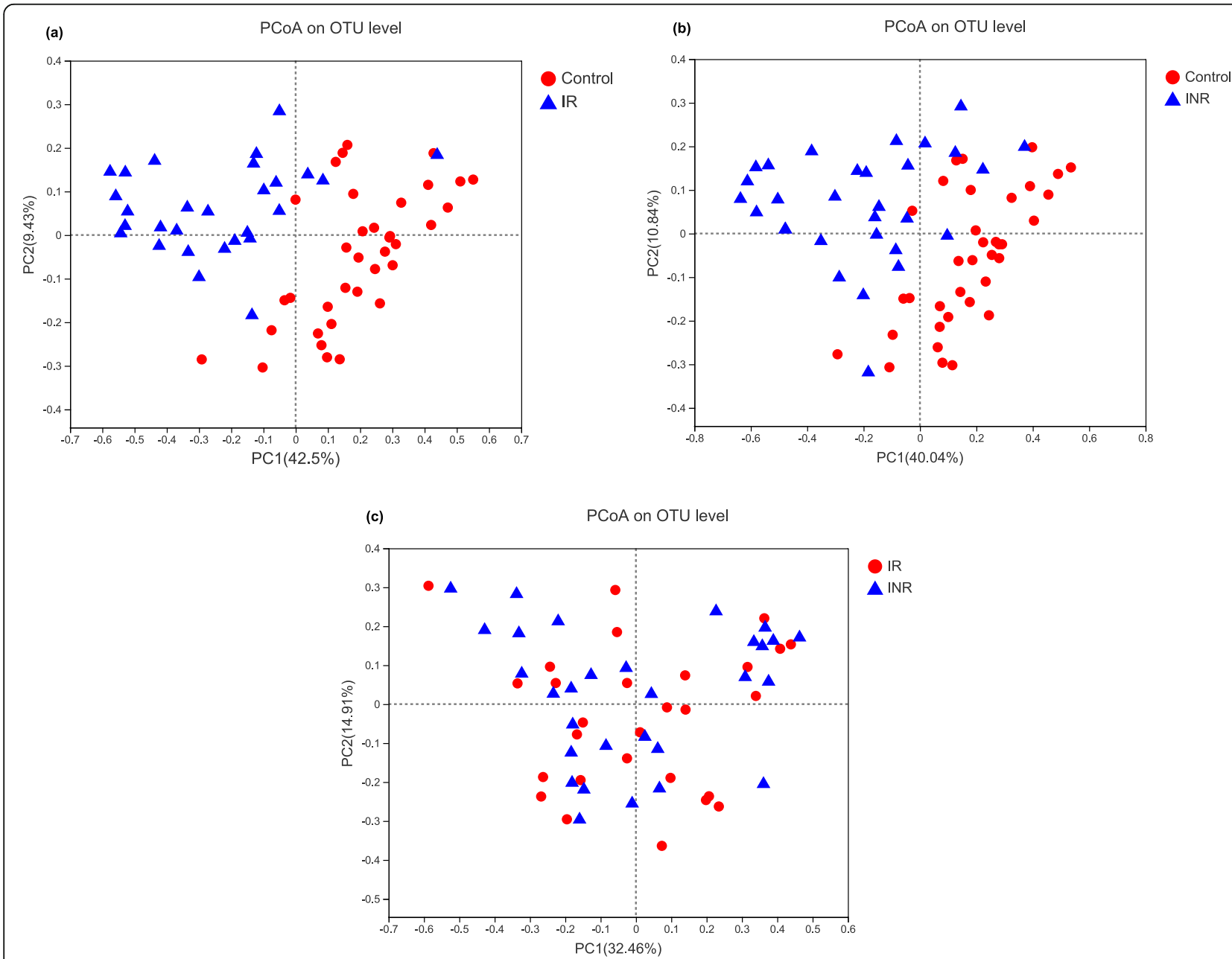

Fig. 1 Principal coordinates analysis (PCOA) of microbiomes in the patients and the healthy controls. Remarkable differences of bacterial communities among the immunological responders (IR), immunological non-responders (INR) and the healthy controls (a, b); no significant difference between the IR and INR groups (c) 


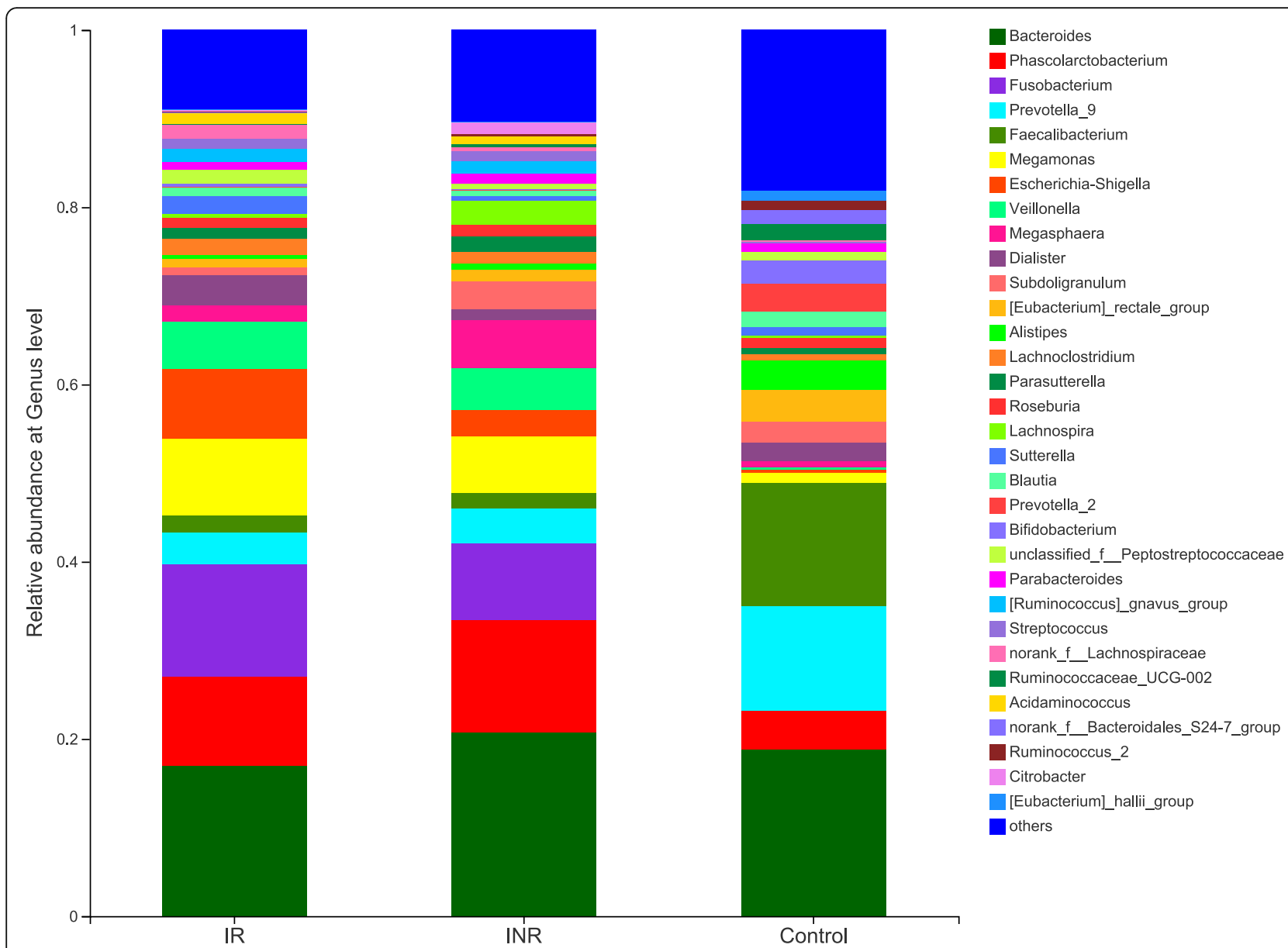

Fig. 2 The relative abundance of fecal microbiota at the genus level in the patients and the healthy controls. IR: immunological responders; INR: immunological non-responders; Control: healthy controls

groups. At the phylum level, Bacteroidetes, Actinobacteria, Lentisphaerae are more abundant in the healthy controls than those in the INR group, while Proteobacteria, Fusobacteria, Tenericutes, Saccharibacteria and unclassified $k$ norank are more abundant in the INR group than those in the healthy controls (Fig. 2 and S1 Fig. 2c). At the genus level, the relative abundances of 83 genera (including 11 predominant genera) are different between the healthy controls and the INR groups. The relative proportions of Faecalibacterium, Eubacterium rectale, Alistipes, Bifidobacterium, Blautia, Roseburia and Ruminococcaceae are more abundant in the healthy controls than those in the INR group. Parasutterella, Megasphaera, Fusobacterium, and Ruminococcus gnavus are found to be overrepresented in the INR group (Fig. 2 and S1 Fig. 2d). Although there is no significant difference between the IR and the INR group at the phylum level (S1 Fig. 2e), the abundances of 12 genera (including 2 predominant genera) are different between the IR and INR groups. The abundances of the two predominant genera Escherichia-Shigella and
Blautia are significantly higher in the IR than those in the INR group (Fig. 2 and S1 Fig. 2f).

In order to identify the key phylotypes responsible for the difference found in distinguishing the fecal microbiota of different groups, linear discriminant analysis (LDA) effect size (LEfSe) was performed and a threshold of three on effect size was used. Taxonomic cladograms which represents the microbiota structure and predominant bacteria in the three groups is presented, and the biggest differences between the two communities are presented in S4 Fig.

\section{Comparison of the T-cell activation in the IR and INR groups}

As expected, nadir CD4+ T-cell, current CD4+ T-cell counts and CD4/CD8 ratio are lower in the INR group than those in the IR group $(p<0.0001)$. The proportion of CD8 + CD57+ T-cell in the INR group is significantly lower than those in the IR group $(p<0.001)$. The proportion level of $\mathrm{CD} 4+$ and $\mathrm{CD} 8+\mathrm{T}$-cells immune 
activation (CD4/8+ T-cell by the expression of CD25+, HLA-DR+, and HLA-DR+/CD38+) is similar in the INR and IR groups (Table 1 ).

\section{Comparison of the bacterial translocation markers and inflammation profiles}

Lipopolysaccharide (LPS), which translocates from the gut to the blood stream, is commonly used as the major antigens to drive the chronic immune activation. The level of LPS is significantly higher in the INR group compared with other groups $(p<0.0001)$. However, the soluble immune activation marker sCD14 shows no difference between the groups. Of the 13 markers studied, the level of IL-13 shows no difference among groups while the other 12 markers (IL-2, IL-4, IL-5, IL-6, IL-9, IL10, IL-17A, IL-17F, IL-21, IL-22, IFN- $\gamma$ and TNF- $\alpha$ ) appear to be significantly higher in the INR and IR groups when compared with the healthy controls, but there is no significant difference between the INR and IR groups (Table 1).

\section{Association between fecal microbiota and immune activation}

Spearman correlations of the relative abundances of bacteria genera and levels of T-cell activation, inflammation or translocation biomarkers are evaluated (Fig. 3). Interestingly, nadir CD4+ T-cell counts are positively correlated with the abundances of Ruminococcaceae and Alistipes, while current CD4+ T-cell counts are strongly positively correlated with the abundances of Ruminococcaceae and Subdoligranulum. The genus Fusobacterium is negatively correlated with nadir and current CD4+ Tcell. The CD4/CD8 ratio is positively correlated with the genera Faecalibacterium and Ruminococcaceae, but negatively correlates with Escherichia-Shigella. Moreover, the CD8 + CD57+ T-cell counts is positively correlated with Escherichia-Shigella but negatively correlates with the genera Ruminococcaceae and Alistipes. The genera Roseburia and Blautia are negatively associated with nadir $\mathrm{CD} 4+\mathrm{T}$-cell and positively associated with CD8 + CD57+ T-cell counts. Inflammation markers and LPS are positively correlated with the Ruminococcus and

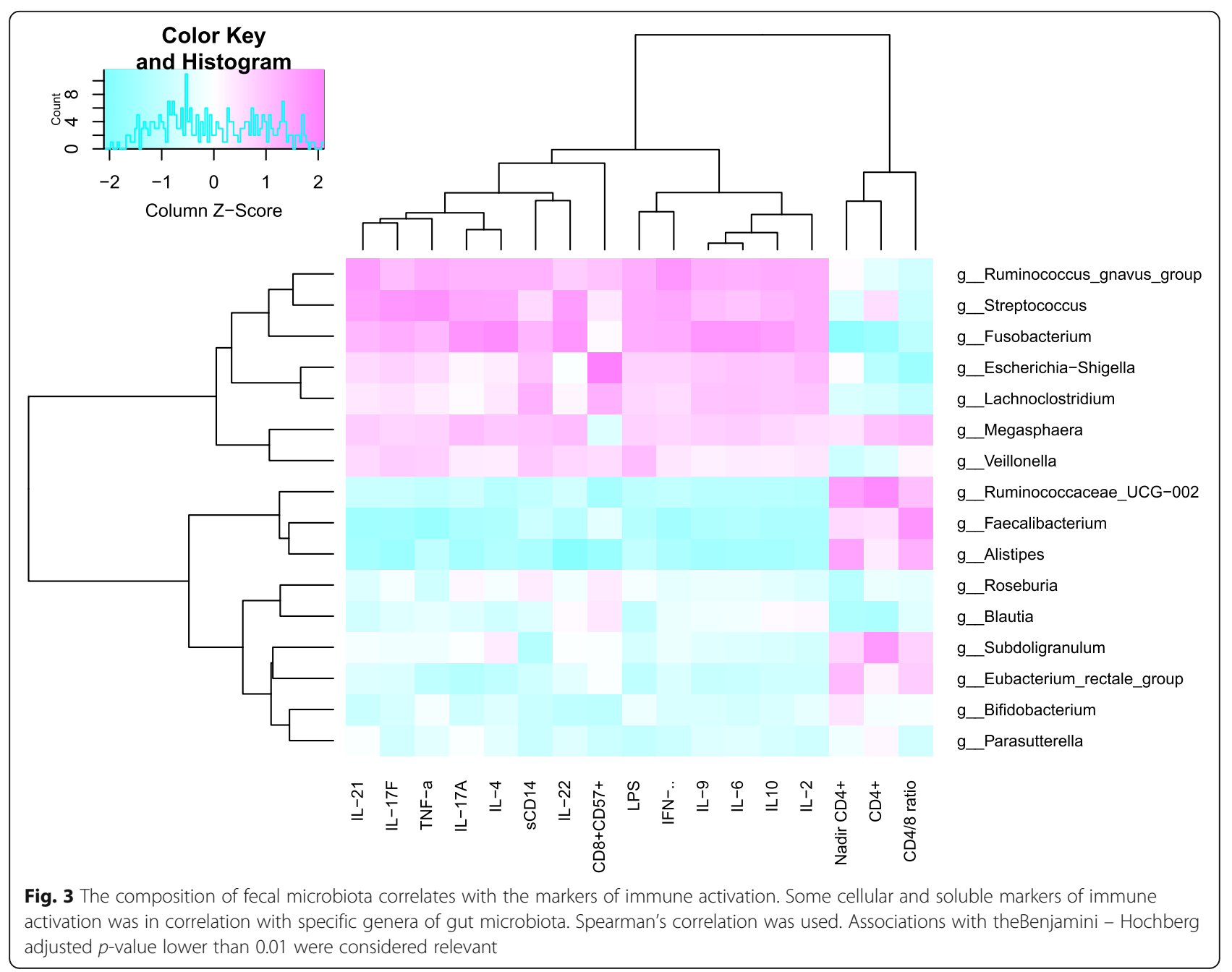


Fusobacterium but negatively correlates with the genus Faecalibacterium (Fig. 3).

\section{Discussion}

The objective of highly active antiretroviral therapy (HAART) is to achieve immune reconstitution and successful viral suppression in HIV-infected patients [2]. Even with complete viral suppression, the CD4+ T cell cannot be reversed completely and microbial translocation continues after peripheral CD4+ T cell restoration [20-23]. Gut microbiota has been reported to have critical impact on human biology and pathophysiology. The gut microbiota's function as a key factor in the process of immune homeostasis is accepted widely by the research community now [23]. Compositional and functional changes of the gut microbiota have indicated a new relationship between the gut bacterial community and immunity in HIV-infected individuals with treatments [7, 24-26]. However, the way the microbiome contributes to immune response in HIV positive individuals is poorly understood. So, the objective of this study is to investigate the relationship between the fecal microbiome and immune activation in HIV-positive individuals with different immune responses to long-term ART in China.

The microbiome dysbiosis in HIV-infected individuals has been introduced in several studies $[7,25$, 26]. However, early studies only adopted a small amount of IR and INR patients as subjects $[8,10]$ and did not taken immune response to HAART into consideration [27]. The previous studies showed that the relative abundance of Fusobacterium was independently associated with poorer $\mathrm{CD} 4+\mathrm{T}$-cell recovery [10], and the relative abundances of unclassified Subdoligranulum $s p$. and C. comes were positively correlated with CD8 + HLA-DR+ T-cell counts and CD8 + HLADR+/CD8+ percentage in the HIV-infected subjects [8]. The current study has adopted higher number of IR and INR patients and have expanded the range of observations when investigating the relationship between the gut microbiota and immune activation. This study found that the ecological indices of microbiota (including community diversity, richness and observed species numbers) were significantly lower in the IR and INR groups when compared with the healthy controls. Additionally, PCoA analysis showed remarkable differentiation of bacterial communities between the IR, INR and the healthy controls. The findings suggested that HIV-infected immunological responders and immunological nonresponders could not recover completely from the gut microbiota dysbiosis.

Furthermore, this study found that the IR and INR group had a unique bacterial signature at the phylum level. Proteobacteria, Fusobacteria, and Saccharibacteria were more abundant, whereas Bacteroidetes, Actinobacteria and Lentisphaerae were depleted in the IR and INR groups. At the $97 \%$ similarity level, the predominant genera Fusobacterium, Ruminococcus gnavus and Megamonas were more abundant, whereas Faecalibacterium, Alistipes, Bifidobacterium, Eubacterium rectale, and Roseburia were depleted in the IR and INR groups compared to the healthy controls. Different taxa from bacterial phyla Proteobacteria, Fusobacteria and Bacteroidetes between HIV positive and negative individuals have been reported in related studies. The result of this study indicates that the Proteobacteria were more abundant in HIV-infected individuals, which is consistent with most of the published studies, while other studies report no change [8, 10-12, 28-33]. The family Enterobacteriaceae was reported positively associated with markers of monocyte activation (sCD14), inflammation and colonic $\mathrm{T}$ cell activation and inversely correlated with blood CD4+ T-cell counts [30, 32, 34]. Escherichia-Shigella, which belonged to Enterobacteriaceae, were negatively correlated with the CD4/CD8 ratio, but positively correlated with the CD8 + CD57+ T-cell which was the hallmark of immunosenescence in HIV infection [35, 36]. Furthermore, Escherichia-Shigella was more abundant in the IR group than that in INR group in this study.

The phylum Fusobacteria (mostly driven by its constituent genus Fusobacterium) was reported to be associated with intestinal inflammation [37, 38] and was abundant in HIV-infected individuals in some studies $[29,33,39,40]$. Here, this study found increased abundance of Fusobacterium in the IR and INR groups. Furthermore, inflammation markers and translocation biomarkers (LPS) were positively correlated with Fusobacterium, while the nadir and current CD4+ T-cell counts were negatively correlated with Fusobacterium in this study. This was consistent with the previous published studies which reported that the relative abundance of Fusobacterium was independently associated with poorer CD4 T-cell recovery and the enrichment of Fusobacterium was associated with decreased ability of immune recovery and persistent immune dysfunction following ART [10].

The phylum Bacteroidetes, which include the families Bacteroidaceae, Prevotellaceae, Porphyromonadaceae and Rikenellaceae, exhibited a more heterogeneous pattern of changes in the HIV-infected individuals [11, 12, 23, 28-34, 39-42]. The family Bacteroidaceae (mostly driven by the abundance of the genus Bacteroides) which was generally considered to be playing an antiinflammatory role [43-46], was depleted in the IR and INR groups. The bile-tolerant family Rikenellaceae (mostly driven by the abundance of the genus Alistipes) which had protective properties against $C$. difficile 
infection [46, 47], was overall depleted in the IR and INR groups. Actinobacteria, which was reported to be similar in the proximal gut of HIV-infected patients and negative controls [32], were depleted in the IR and INR groups in this study. The Alistipes was negatively correlated with the CD8 + CD57+ T-cell and positively correlated with the nadir $\mathrm{CD} 4+\mathrm{T}$-cell counts. Notably, the commonly used probiotics Bifidobacterium which belong to the phylum of Actinobacteria was depleted in the IR and INR groups compared with the healthy controls [48].

Although the abundance of phylum Firmicutes was similar in all three groups, genera such as Faecalibacterium and Ruminococcaceae were depleted in the IR and INR groups compared with the healthy controls. Ruminococcaceae has been playing both protective and disruptive roles within the gut microbial community, such as the production of anti-inflammatory short-chain fatty acids (SCFA) [49] or the degradation of host mucus and potential proinflammatory in IBD [50]. In this study, Faecalibacterium and Ruminococcaceae UCG-002, which belong to the family of Ruminococcaceae were depleted in the IR and INR groups compared with the healthy controls. Interestingly, Ruminococcaceae was positively correlated with the current nadir $\mathrm{CD} 4+\mathrm{T}$-cell counts while negatively correlated with the CD8 + CD57+ T-cell counts. In addition, inflammation markers and LPS were positively correlated with the abundance of genus Ruminococcus. Faecalibacterium has been reported as the anti-inflammatory commensal genus $[29,51]$. In this study, the genus Faecalibacterium was positively correlated with $\mathrm{CD} 4 / \mathrm{CD} 8$ ratio while negatively correlated with the inflammation markers and LPS.

The normal (gut microbiota) groups which commonly present in large amounts in the healthy controls bear more significance and interest us more compared to various pathogens. Both Roseburia and Blautia (which belong to the phylum Firmicutes, class Clostridia, family Lachnospiraceae) were members of the groups of commensals like which described above and have been described as the SCFA producers with beneficial effect on the intestinal barrier and an important energy source for epithelial cells [52]. In this study, Blautia, and Roseburia were more abundant in the healthy controls and the abundance of Blautia increased significantly in the IR group than the INR group. Roseburia and Blautia were negatively associated with the nadir $\mathrm{CD} 4+\mathrm{T}$-cell and positively associated with the CD8 + CD57+ T-cell counts. These suggested that Blautia and Roseburia might be associated with the treatment outcome.

Altogether, these results indicate that immune activation in the HIV-infected patients was associated with the gut microbiota dysbiosis observed. Based upon these findings, this study speculate that the gut microbiota may be one of the factors contributing to different immune responses to HAART. Fusobacterium, Alistipes, Ruminococcaceae, Faecalibacterium, Escherichia-Shigella, Roseburia and Blautia maybe the major genera contributing to different immune responses and treatment outcome in immunodiscordant and immunoconcordant patients with long-term suppressive ART. However, we did not collect samples before the ART treatment, so future research on whether the gut microbiome composition influences treatment effect or not is still needed and this study cannot directly address the question if changes in the microbiota were causative or rather a result of systemic HIV-1associated immune activation. This study acknowledges that the extensive dietary data and various living conditions of the subjects involved may lead to biases during the analysis. On the other hand, while correlation analysis was helpful in linking biological clues to the impact of dysbiosis in immune responses in the patients, a direct manipulation of the microbiome was needed to validate their cellular and biochemical actions in vitro or in vivo, and the exact mechanism of how HIV infection can lead to dysbiosis in the gut need to be studied in the future.

\section{Conclusions}

In summary, this study presents the research results regarding the gut microbiota dysbiosis in HIV-infected immunological non-responders and immunological responders, and concludes that the gut microbiota dysbiosis may be one of the factors contributing to different immune responses to HAART. Fusobacterium, Alistipes, Ruminococcaceae, Faecalibacterium, Escherichia-Shigella, Roseburia and Blautia may be the major genera contributing to different immune responses and treatment outcome in immunodiscordant and immunoconcordant patients with long-term suppressive ART.

\section{Methods \\ Recruitment of subjects}

The process of participants' recruitment and sample collection is stated in Suppl. S5 Fig. 5. 8 HIV-infected individuals in total, including 28 immunological responders (IR), 30 immunological non-responders (INR), and 36 healthy subjects (healthy controls) were recruited. IR and INR were defined as patients whose CD4+ T-cell counts/ $\mu \mathrm{l}$ equal or is more than 500 or less than 200 after 2 years of receiving complete viral suppression therapy respectively. Subjects were recruited from the HIV clinic of the First Affiliated Hospital of Zhejiang University from November 2015 to October 2017. All HIV-positive participants were diagnosed by the Disease Control and Prevention Center of Zhejiang Province. All HIV-positive subjects were on two nucleoside reverse transcriptase inhibitors (NRTIs) + nonnucleoside reverse transcriptase inhibitors (NNRTIs) or the protease 
inhibitor-based therapy: Zidovudine/Tenofovir Disoproxil Fumarate (AZT/TDF) + Lamivudine $\quad(3 \mathrm{TC})+$ Efavirenz (EFV) or Lopinavir/ritonavir (LPV/r). The healthy controls were all healthy HIV-negative volunteers and most of them belong to the staff of the institution. Age, gender, and body mass index (BMI) of the healthy controls are similar to those of the HIV-positive individuals (Table 1). Candidates with following conditions and traits are excluded from the subject selection: age over 60 years old; having opportunistic infection; having hepatitis $\mathrm{B}$ or $\mathrm{C}$ infection; having history of using antibiotics, immunosuppressive regimen, probiotics, prebiotics, or symbiotics in the previous 6 months; having used rectally administered medications within $48 \mathrm{~h}$ before selection; $\mathrm{BMI}>30$; having a history of inflammatory bowel disease (IBD); having active inflammation affecting the gastro intestines.

\section{Ethics statement}

All participants provided written informed consents before participating in the study. This study conforms to the ethical norms of the 1975 Helsinki Declaration. The research protocol was approved by the Institutional Review Committee of the First Affiliated Hospital of Zhejiang University on October 7, 2015. All the data used for analysis were anonymized.

\section{Fecal samples collection and DNA extraction}

Fecal samples of the participants were collected in sterile container before their clinic visits and were stored in a $80^{\circ} \mathrm{C}$ environment before the DNA extraction. The DNA was extracted using a QIAamp DNA stool mini kit (QIAGEN, Hilden, Germany) and glass-bead beat on Mini-bead beater (FastPrep; Thermo Electron Corporation, Boston, MA, USA), following the manufacturer's instructions. The DNA's quantification and purity were assessed by NanoDrop ND-1000 spectrophotometer (Thermo Electron Corporation). The integrity and sizes of the DNA were reviewed by $1.0 \%$ agarose gel electrophoresis. The DNA was stored at a $-20^{\circ} \mathrm{C}$ environment for further analysis.

\section{Polymerase chain reaction (PCR) and 16S rRNA gene sequencing} PCR amplification of the bacterial 16S rRNA gene V3$\mathrm{V} 4$ region was performed using universal primers $(338 \mathrm{~F}$ 5' - ACTCCTACGGGAGGCAGCAG-3', 806R5'-GGAC TACHVGGGTWTCTAAT-3'). The PCR reactions were conducted using the following program: 3 mins of denaturation at $95^{\circ} \mathrm{C}, 27$ cycles of 30 s at $95^{\circ} \mathrm{C}, 30$ s for annealing at $55^{\circ} \mathrm{C}$, and $45 \mathrm{~s}$ for elongation at $72{ }^{\circ} \mathrm{C}$, and a final extension at $72{ }^{\circ} \mathrm{C}$ for 10 mins. PCR reactions were performed in a triplicate $20 \mu \mathrm{l}$ mixture containing $4 \mu \mathrm{l}$ of $5 \times$ FastPfu Buffer, $2 \mu \mathrm{l}$ of $2.5 \mathrm{mM}$ dNTPs, $0.8 \mu \mathrm{l}$ of each primer $(5 \mu \mathrm{M}), 0.4 \mu \mathrm{l}$ of FastPfu Polymerase and $10 \mathrm{ng}$ of the template DNA. The resulted PCR products were extracted from a $2 \%$ agarose gel and then purified using the AxyPrep DNA Gel Extraction Kit (Axygen Biosciences, Union City, CA, USA) and quantified using QuantiFluor $^{\mathrm{TM}}$-ST (Promega, USA) following the manufacturer's protocol. Purified amplicons were pooled in equimolar and paired-end sequenced $(2 \times 300)$ on an Illumina MiSeq platform (Illumina, San Diego, USA) following the standard protocols by Majorbio Bio-Pharm Technology Co. Ltd. (Shanghai, China). The raw reads were deposit into the NCBI Sequence Read Archive (SRA) database and is accessible with the following link: https://www.ncbi.nlm.nih.gov/sra/PRJNA533202.

\section{Bioinformatics and statistics}

The raw fastq files were demultiplexed and qualityfiltered using Trimmomatic, and then merged by FLAS $H$. The original reads were trimmed with a minimum length of $50 \mathrm{bp}$ with an average quality score of 20 . Two mismatches were allowed in primer sequences and reads containing ambiguous bases were removed. Ten homopolymers authorized in sequences were limited. OTUs were picked at a $97 \%$ similar threshold by UPARSE (http://drive5.com/uparse/) and chimera identification sequence was performed using UCHIME. Taxonomybased analyses were performed by RDP Classifier algorithm (http://rdp.cme.msu.edu/) in combination with 16 s rRNA Silva128 database with $70 \%$ cut-off confidence.

\section{Viral load, flow Cytometry and Immunophenotype}

Quantifications of CD4+ and CD8+ T-cells as well as HIV-1 RNA were carried out in HIV-infected individuals using flow cytometry and Cobas Amplicor (Roche Molecular Systems Inc., Branchburg, New Jersey, USA) as the clinical routine. The percentage of CD4+ and CD8+ $\mathrm{T}$ cells expressing markers of activation (CD25+, CD38+, HLADR+, or CD38+/HLA-DR+) and senescence $(\mathrm{CD} 57+)$ were quantified by the BD FACS Canto II flow cytometer (BD Biosciences, California, USA) using fresh anticoagulated whole blood. Antibody such as CD3-FITC, CD4- PerCP/Cy5.5, CD8-Brilliant Violet $510^{\mathrm{Tm}}$, CD38-Brilliant Violet 421, CD25-PE, HLA-DR$\mathrm{APC}_{\text {Fire }}^{\mathrm{Tm}}$ 750, and CD57-allophycocyanin (APC) were purchased from Biolegend (San Diego, CA).

\section{Bacterial translocation and immune activation markers}

Sera samples of 27 IR, 30 INR, and 17 healthy participants were collected for the measurement of the immune activation markers. These markers were quantified using LEGE NDplex $^{\text {Tx }}$ Human Th Cytokine Panel (Biolegend, San Diego, CA): IL-2, IL-4, IL-6, IL-9, IL10, IL-13, IL-17A, IL17F, IL-21, IL-22, TNF- $\alpha$ and interferon (IFN)- $\gamma$, in line with the manufacturer's instructions. Human Lipopolysaccharides (LPS) ELISA Kit (CUSABIO; Wuhan, China) and 
Human soluble CD14 (sCD14) ELISA Kit (MultiSciences, Hangzhou, China) were used to test the plasma LPS and sCD14 following the standard protocols. Two replicates were performed for each assay.

\section{Statistics analysis}

OTUs that reached the $97 \%$ level of nucleotide similarity level were used for alpha diversity (Shannon, Simpson, and Sobs), richness (ACE and Chao1), Good's coverage, and rarefaction curve, and phylogenetic beta diversity measures analyses by mothur. PERMANOVA (permutational multivariate analysis of variance) were used to assess beta diversity based on the UniFrac distances. The number of permutations was 999 for PERMANOVA. The results were imported into Phyloseq for subsampling normalization, manipulation, and graph visualization by $\mathrm{R}$ (V.3.1.3, The R Project for Statistical Computing) [53]. Linear discriminant analysis (LDA) effect size (LEfSe) used the Kruskal-Wallis rank sum test to detect features with significant different abundances between the assigned taxa. LEfSe is available online in the Galaxy workflow framework [54]. Principal Coordinates analysis (PCoA) was conducted for weighted Unifrac data to visualize the microbial communities. The one-way ANOVA, nonparametric test, Wilcoxon rank sum test and MannWhitney $U$ test which used for comparisons between groups were conducted in the R package and SPSS software (version 21, SPSS, Inc., Chicago, IL, USA). The average abundance values for each bacterium are depicted as mean \pm SD. A significant alpha of 0.05 and LDA effect size threshold of 3 were used for all biomarkers. Correlations between the variables were calculated using the Spearman's rank-correlation analysis by $\mathrm{R}$ package; and associations with the Benjamini-Hochberg adjusted $p$-value lower than 0.01 were considered relevant.

\section{Supplementary Information}

The online version contains supplementary material available at https://doi. org/10.1186/s12866-020-02074-1.

Additional file 1: Figure S1. Taxonomic differences of fecal microbiota between the patients and healthy controls groups. Comparison of relative abundances at the bacterial phylum $(a, c, e)$ and genus $(b, d, f)$ levels between the immunological responders (IR), immunological nonresponders (INR) and the healthy controls (Control) group. \# indicates $P<$ 0.05. * indicates $P<0.01$. The average abundance values for each bacterium are depicted as mean \pm SD.

Additional file 2: Figure S2. The relative abundance bar chart figure of each sample at the phylum level. IR: immunological responders; INR: immunological non-responders; Control: healthy controls.

Additional file 3: Figure S3. The relative abundance bar chart figure of each sample at the genus level. IR: immunological responders; INR: immunological non-responders; Control: healthy controls.

Additional file 4: Figure S4. Taxonomic differences of fecal microbiota between the immunological responders (IR), immunological nonresponders (INR) and healthy controls (Control) group. Cladogram representing the features that are discriminative using the LDA model results on the bacterial hierarchy $(a, c, e)$. LDA coupled with effect size measurements identifies the most differentially abundant taxon between the two groups $(b, d, f)$.

Additional file 5: Figure S5. The recruitment of participants and the process of sample collection.

Additional file 6: Table S1. The fecal microbiota relative abundance of each sample in different groups at the genus level.

\section{Abbreviations}

HIV: Human immunodeficiency virus; ART: Antiretroviral therapy; HAAR T: Highly active antiretroviral therapy; INR: Immunological non-responders; IR: Immunological responders; BMI: Body mass index; MSM: Men who have sex with men; SCFA: Short-chain fatty acids; LPS: Lipopolysaccharide; PCoA: Principal coordinate analysis; LDA: Linear discriminant analysis; LEfSe: Linear discriminant analysis effect size; OTU: Operational taxonomic unit; $16 \mathrm{~S}$ rRNA: $16 \mathrm{~S}$ ribosomal RNA; NRTIs: Nucleoside reverse transcriptase inhibitors; NNRTIs: Nonnucleoside reverse transcriptase inhibitors;

AZT: Zidovudine; TDF: Tenofovir Disoproxil Fumarate; 3TC: Lamivudine; EFV: Efavirenz; LPV/r: Lopinavir/ritonavir

\section{Acknowledgments}

This study gratefully acknowledges the patients who participated in the research.

\section{Authors' contributions}

YX participated in the designing of the study and performed the statistical analysis and wrote the paper. JS performed the statistical analysis and revised the paper. $\mathrm{LW}, \mathrm{HJ}, \mathrm{CH}$ and $\mathrm{JY}$ carried out the experiment. $\mathrm{CH}$ and $\mathrm{YH}$ collected the biopsy samples. BR helped to review the manuscript. BZ participated in the designing and reviewing of the manuscript. All authors read through and approved the final manuscript.

\section{Funding}

This study is funded by the Natural Science Foundation of China (Young Scientist Fund, 81500491) and the National Science Foundation of China (Major Research Plan, 2018ZX10715-014). These funding agencies have no role in the design, data collection, analysis or interpretation of the research or in the writing of the manuscript.

\section{Availability of data and materials}

The raw reads were deposited into the NCBI Sequence Read Archive (SRA) database and is accessible with the following link: https://www.ncbi.nlm.nih. gov/sra/PRJNA533202

\section{Ethics approval and consent to participate}

This study conforms to the ethical norms of the 1975 Helsinki Declaration. The research protocol was approved by the Institutional Review Committee of the First Affiliated Hospital of Zhejiang University on October 7, 2015. All data analyzed were anonymized. All participants provided a written informed consent before entering the study.

Consent for publication

Not applicable.

\section{Competing interests}

The authors declare that they have no conflict of interests. Informed consent: Written consent was obtained from the patients.

\section{Author details}

${ }^{1}$ State Key Laboratory for Diagnosis and Treatment of Infectious Diseases, National Clinical Research Center for Infectious Diseases, Collaborative Innovation Center for Diagnosis and Treatment of Infectious Diseases, The Department of Infectious Diseases, The First Affiliated Hospital, School of Medicine, Zhejiang University, 79, QingChun Road, Hangzhou 310003, China. ${ }^{2}$ Ningbo Medical Center Lihuili Hospital, Ningbo 315000, China. 
Received: 15 July 2020 Accepted: 16 December 2020

Published online: 06 January 2021

\section{References}

1. Kelley CF, Kitchen CM, Hunt PW, Rodriguez B, Hecht FM, Kitahata M, Crane HM, Willig J, Mugavero M, Saag M, et al. Incomplete peripheral CD4+ cell count restoration in HIV-infected patients receiving long-term antiretroviral treatment. Clin Infect Dis. 2009;48(6):787-94.

2. Cenderello G, De Maria A. Discordant responses to CART in HIV-1 patients in the era of high potency antiretroviral drugs: clinical evaluation, classification, management prospects. Expert Rev Anti-Infect Ther. 2016;14(1):29-40.

3. Tsukamoto H, Clise-Dwyer K, Huston GE, Duso DK, Buck AL, Johnson LL, Haynes L, Swain SL. Age-associated increase in lifespan of naive CD4 T cells contributes to T-cell homeostasis but facilitates development of functional defects. Proc Natl Acad Sci U S A. 2009;106(43):18333-8.

4. Florence E, Lundgren J, Dreezen C, Fisher M, Kirk O, Blaxhult A, Panos G, Katlama C, Vella S, Phillips A, et al. Factors associated with a reduced CD4 lymphocyte count response to HAART despite full viral suppression in the EuroSIDA study. HIV Med. 2003;4(3):255-62.

5. Kaufmann GR, Perrin L, Pantaleo G, Opravil M, Furrer H, Telenti A, Hirschel B,

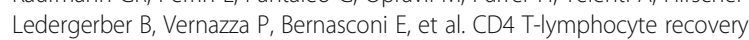
in individuals with advanced HIV-1 infection receiving potent antiretroviral therapy for 4 years: the Swiss HIV cohort study. Arch Intern Med. 2003; 163(18):2187-95

6. Gazzola L, Tincati C, Bellistri GM, Monforte A, Marchetti G. The absence of CD4+ T cell count recovery despite receipt of virologically suppressive highly active antiretroviral therapy: clinical risk, immunological gaps, and therapeutic options. Clin Infect Dis. 2009;48(3):328-37.

7. Gootenberg DB, Paer JM, Luevano JM, Kwon DS. HIV-associated changes in the enteric microbial community: potential role in loss of homeostasis and development of systemic inflammation. Curr Opin Infect Dis. 2017;30(1):3143.

8. Lu W, Feng Y, Jing F, Han Y, Lyu N, Liu F, Li J, Song X, Xie J, Qiu Z, et al. Association between gut microbiota and CD4 recovery in HIV-1 infected patients. Front Microbiol. 2018;9:1451.

9. Ji Y, Zhang F, Zhang R, Shen Y, Liu L, Wang J, Yang J, Tang Q, Xun J, Qi T, et al. Changes in intestinal microbiota in HIV-1-infected subjects following CART initiation: influence of CD4+ T cell count. Emerg Microbes Infect. 2018; $7(1): 113$.

10. Lee SC, Chua LL, Yap SH, Khang TF, Leng CY, Raja Azwa Rl, Lewin SR, Kamarulzaman A, Woo YL, Lim YAL, et al. Enrichment of gut-derived Fusobacterium is associated with suboptimal immune recovery in HIVinfected individuals. Sci Rep. 2018:8(1):14277.

11. Sun Y, Ma Y, Lin P, Tang YW, Yang L, Shen Y, Zhang R, Liu L, Cheng J, Shao $J$, et al. Fecal bacterial microbiome diversity in chronic HIV-infected patients in China. Emerg Microbes Infect. 2016:5:e31.

12. Ling Z, Jin C, Xie T, Cheng Y, Li L, Wu N. Alterations in the fecal microbiota of patients with HIV-1 infection: an observational study in a Chinese population. Sci Rep. 2016;6:30673.

13. Deeks SG, Tracy R, Douek DC. Systemic effects of inflammation on health during chronic HIV infection. Immunity. 2013;39(4):633-45.

14. West $C E$, Renz H, Jenmalm MC, Kozyrskyj AL, Allen KJ, Vuillermin P, Prescott SL. The gut microbiota and inflammatory noncommunicable diseases: associations and potentials for gut microbiota therapies. J Allergy Clin Immunol. 2015:135(1):3-13 quiz 14.

15. Klatt NR, Chomont N, Douek DC, Deeks SG. Immune activation and HIV persistence: implications for curative approaches to HIV infection. Immunol Rev. 2013;254(1):326-42.

16. Klatt NR, Funderburg NT, Brenchley JM. Microbial translocation, immune activation, and HIV disease. Trends Microbiol. 2013;21(1):6-13.

17. Lederman MM, Funderburg NT, Sekaly RP, Klatt NR, Hunt PW. Residual immune dysregulation syndrome in treated HIV infection. Adv Immunol. 2013;119:51-83.

18. Siedner MJ. START or SMART? Timing of Antiretroviral Therapy Initiation and Cardiovascular risk for people with human immunodeficiency virus infection. Open Forum Infect Dis. 2016:3(1):ofw032.

19. Havlir DV, Currier JS. CROI 2015: complications of HIV infection and antiretroviral therapy. Top Antivir Med. 2015;23(1):56-65.

20. Jiang W, Lederman MM, Hunt P, Sieg SF, Haley K, Rodriguez B, Landay A, Martin J, Sinclair E, Asher Al, et al. Plasma levels of bacterial DNA correlate with immune activation and the magnitude of immune restoration in persons with antiretroviral-treated HIV infection. J Infect Dis. 2009;199(8): 1177-85.

21. Mavigner M, Cazabat M, Dubois M, L'Faqihi FE, Requena M, Pasquier C, Klopp P, Amar J, Alric L, Barange K, et al. Altered CD4+ T cell homing to the gut impairs mucosal immune reconstitution in treated HIV-infected individuals. J Clin Invest. 2012;122(1):62-9.

22. Neuhaus J, Jacobs DR Jr, Baker JV, Calmy A, Duprez D, La Rosa A, Kuller LH, Pett SL, Ristola M, Ross MJ, et al. Markers of inflammation, coagulation, and renal function are elevated in adults with HIV infection. J Infect Dis. 2010; 201(12):1788-95.

23. Vazquez-Castellanos JF, Serrano-Villar S, Latorre A, Artacho A, Ferrus ML, Madrid N, Vallejo A, Sainz T, Martinez-Botas J, Ferrando-Martinez S, et al. Altered metabolism of gut microbiota contributes to chronic immune activation in HIV-infected individuals. Mucosal Immunol. 2015;8(4):760-72.

24. Cho I, Blaser MJ. The human microbiome: at the interface of health and disease. Nat Rev Genet. 2012;13(4):260-70.

25. Lujan JA, Rugeles MT, Taborda NA. Contribution of the microbiota to intestinal homeostasis and its role in the pathogenesis of HIV-1 infection. Curr HIV Res. 2019;17(1):13-25.

26. Vujkovic-Cvijin I, Somsouk M. HIV and the gut microbiota: composition, consequences, and avenues for amelioration. Curr HIV AIDS Rep. 2019;16(3): 204-13.

27. Tincati C, Merlini E, Braidotti P, Ancona G, Savi F, Tosi D, Borghi E, Callegari ML, Mangiavillano B, Barassi A, et al. Impaired gut junctional complexes feature late-treated individuals with suboptimal CD4+ T-cell recovery upon virologically suppressive combination antiretroviral therapy. AIDS. 2016;30(7): 991-1003.

28. Lozupone CA, Rhodes ME, Neff CP, Fontenot AP, Campbell TB, Palmer BE. HIV-induced alteration in gut microbiota: driving factors, consequences, and effects of antiretroviral therapy. Gut Microbes. 2014;5(4):562-70.

29. Mutlu EA, Keshavarzian A, Losurdo J, Swanson G, Siewe B, Forsyth C, French A, Demarais P, Sun Y, Koenig L, et al. A compositional look at the human gastrointestinal microbiome and immune activation parameters in HIV infected subjects. PLoS Pathog. 2014;10(2):e1003829.

30. Vujkovic-Cvijin I, Dunham RM, Iwai S, Maher MC, Albright RG, Broadhurst MJ, Hernandez RD, Lederman MM, Huang Y, Somsouk M, et al. Dysbiosis of the gut microbiota is associated with HIV disease progression and tryptophan catabolism. Sci Transl Med. 2013;5(193):193ra191.

31. Dillon SM, Lee EJ, Kotter CV, Austin GL, Dong Z, Hecht DK, Gianella S, Siewe B, Smith DM, Landay AL, et al. An altered intestinal mucosal microbiome in HIV-1 infection is associated with mucosal and systemic immune activation and endotoxemia. Mucosal Immunol. 2014;7(4):983-94.

32. Yang L, Poles MA, Fisch GS, Ma Y, Nossa C, Phelan JA, Pei Z. HIV-induced immunosuppression is associated with colonization of the proximal gut by environmental bacteria. AIDS. 2016;30(1):19-29.

33. Dubourg G, Lagier JC, Hue S, Surenaud M, Bachar D, Robert C, Michelle C, Ravaux I, Mokhtari S, Million M, et al. Gut microbiota associated with HIV infection is significantly enriched in bacteria tolerant to oxygen. BMJ Open Gastroenterol. 2016;3(1):e000080.

34. Dinh DM, Volpe GE, Duffalo C, Bhalchandra S, Tai AK, Kane AV, Wanke CA, Ward HD. Intestinal microbiota, microbial translocation, and systemic inflammation in chronic HIV infection. J Infect Dis. 2015;211(1):19-27.

35. Appay $\vee$, Sauce D. Immune activation and inflammation in HIV-1 infection: causes and consequences. J Pathol. 2008;214(2):231-41.

36. Ivanov II, Frutos Rde L, Manel N, Yoshinaga K, Rifkin DB, Sartor RB, Finlay BB, Littman DR. Specific microbiota direct the differentiation of IL-17-producing T-helper cells in the mucosa of the small intestine. Cell Host Microbe. 2008;4(4):337-49.

37. Nicholson JK, Holmes E, Kinross J, Burcelin R, Gibson G, Jia W, Pettersson S. Host-gut microbiota metabolic interactions. Science. 2012;336(6086):1262-7.

38. Kostic AD, Chun E, Robertson L, Glickman JN, Gallini CA, Michaud M, Clancy TE, Chung DC, Lochhead P, Hold GL, et al. Fusobacterium nucleatum potentiates intestinal tumorigenesis and modulates the tumor-immune microenvironment. Cell Host Microbe. 2013;14(2):207-15.

39. McHardy $\mathrm{HH}_{\text {, Li X }}$, Tong M, Ruegger $\mathrm{P}$, Jacobs J, Borneman J, Anton P, Braun J. HIV infection is associated with compositional and functional shifts in the rectal mucosal microbiota. Microbiome. 2013;1(1):26.

40. Yu G, Fadrosh D, Ma B, Ravel J, Goedert JJ. Anal microbiota profiles in HIVpositive and HIV-negative MSM. AIDS. 2014;28(5):753-60.

41. Nowak P, Troseid M, Avershina E, Barqasho B, Neogi U, Holm K, Hov JR, Noyan K, Vesterbacka J, Svard J, et al. Gut microbiota diversity predicts immune status in HIV-1 infection. AIDS. 2015;29(18):2409-18. 
42. Monaco CL, Gootenberg DB, Zhao G, Handley SA, Ghebremichael MS, Lim ES, Lankowski A, Baldridge MT, Wilen CB, Flagg M, et al. Altered Virome and bacterial microbiome in human immunodeficiency virus-associated acquired immunodeficiency syndrome. Cell Host Microbe. 2016;19(3):311-22.

43. Troy EB, Kasper DL. Beneficial effects of Bacteroides fragilis polysaccharides on the immune system. Front Biosci. 2010;15:25-34.

44. Round JL, Mazmanian SK. Inducible Foxp3+ regulatory T-cell development by a commensal bacterium of the intestinal microbiota. Proc Natl Acad Sc U S A. 2010;107(27):12204-9.

45. Vatanen $T$, Kostic AD, d'Hennezel E, Siljander H, Franzosa EA, Yassour M, Kolde R, Vlamakis $\mathrm{H}$, Arthur TD, Hamalainen AM, et al. Variation in microbiome LPS immunogenicity contributes to autoimmunity in humans. Cell. 2016;165(6):1551.

46. David LA, Maurice CF, Carmody RN, Gootenberg DB, Button JE, Wolfe BE, Ling AV, Devlin AS, Varma Y, Fischbach MA, et al. Diet rapidly and reproducibly alters the human gut microbiome. Nature. 2014;505(7484): 559-63.

47. Khanna S, Montassier E, Schmidt B, Patel R, Knights D, Pardi DS, Kashyap P. Gut microbiome predictors of treatment response and recurrence in primary Clostridium difficile infection. Aliment Pharmacol Ther. 2016;44(7): $715-27$

48. Veiga P, Gallini CA, Beal C, Michaud M, Delaney ML, DuBois A, Khlebnikov A van Hylckama Vlieg JE, Punit S, Glickman JN, et al. Bifidobacterium animalis subsp. lactis fermented milk product reduces inflammation by altering a niche for colitogenic microbes. Proc Natl Acad Sci U S A. 2010;107(42): 18132-7.

49. Flint HJ, Bayer EA, Rincon MT, Lamed R, White BA. Polysaccharide utilization by gut bacteria: potential for new insights from genomic analysis. Nat Rev Microbiol. 2008;6(2):121-31.

50. Png CW, Linden SK, Gilshenan KS, Zoetendal EG, McSweeney CS, Sly LI, McGuckin MA, Florin TH. Mucolytic bacteria with increased prevalence in IBD mucosa augment in vitro utilization of mucin by other bacteria. Am J Gastroenterol. 2010;105(11):2420-8.

51. Shaw KA, Bertha M, Hofmekler T, Chopra P, Vatanen T, Srivatsa A, Prince J, Kumar A, Sauer C, Zwick ME, et al. Dysbiosis, inflammation, and response to treatment: a longitudinal study of pediatric subjects with newly diagnosed inflammatory bowel disease. Genome Med. 2016;8(1):75.

52. Schnorr SL, Candela M, Rampelli S, Centanni M, Consolandi C, Basaglia G, Turroni S, Biagi E, Peano C, Severgnini M, et al. Gut microbiome of the Hadza hunter-gatherers. Nat Commun. 2014;5:3654.

53. McMurdie PJ, Holmes S. phyloseg: an R package for reproducible interactive analysis and graphics of microbiome census data. PLoS One. 2013;8(4): e61217.

54. Goecks J, Nekrutenko A, Taylor J, Galaxy T. Galaxy: a comprehensive approach for supporting accessible, reproducible, and transparent computational research in the life sciences. Genome Biol. 2010;11(8):R86.

\section{Publisher's Note}

Springer Nature remains neutral with regard to jurisdictional claims in published maps and institutional affiliations.

Ready to submit your research? Choose BMC and benefit from:

- fast, convenient online submission

- thorough peer review by experienced researchers in your field

- rapid publication on acceptance

- support for research data, including large and complex data types

- gold Open Access which fosters wider collaboration and increased citations

- maximum visibility for your research: over $100 \mathrm{M}$ website views per year

At $\mathrm{BMC}$, research is always in progress.

Learn more biomedcentral.com/submissions 